Fetal Diagnosis and Therapy
Fetal Diagn Ther 2016;40:123-127

DOI: $10.1159 / 000442154$
Received: July 24, 2015

Accepted: October 30, 2015

Published online: January 21, 2016

\title{
Color Difference in Placentas with Twin Anemia-Polycythemia Sequence: An Additional Diagnostic Criterion?
}

\author{
Lisanne S.A. Tollenaar ${ }^{a}$ Danny P. Zhao ${ }^{b}$ Johanna M. Middeldorp ${ }^{a}$ \\ Femke Slaghekke $^{\mathrm{a}}$ Dick Oepkes $^{\mathrm{a}}$ Enrico Lopriore ${ }^{\mathrm{b}}$ \\ aDivision of Fetal Medicine, Department of Obstetrics and ${ }^{b}$ Division of Neonatology, Department of Pediatrics, \\ Leiden University Medical Centre, Leiden, The Netherlands
}

\author{
Key Words \\ Color difference ratio - Twin anemia-polycythemia \\ sequence $\cdot$ Monochorionic twin placentas
}

\begin{abstract}
Objective: To determine the color intensity difference between the 2 placental shares in monochorionic placentas with twin anemia-polycythemia sequence (TAPS). Methods: We evaluated all digital pictures of TAPS placentas examined at our center and compared them to a control group of uncomplicated monochorionic placentas. We determined the color intensity of individual placental share on the maternal side of each monochorionic placenta using an image processing program and calculated the color difference ratio (CDR). Results: Digital pictures of 19 TAPS and 19 uncomplicated monochorionic placentas were included in this study. The TAPS group consisted of 12 spontaneous TAPS placentas (63\%) and 7 post-laser TAPS placentas (37\%). The median CDR in the group with TAPS was significantly higher than in the control group, 2.73 (range 1.73-6.36) versus 1.09 (range $1.00-1.35)$, respectively $(p<0.01)$. We found a positive correlation between CDR and inter-twin hemoglobin $(\mathrm{Hb})$ difference in the TAPS group $(R=0.66, p<0.01)$ but not in the control group $(R=0.04, p=0.87)$. Conclusion: TAPS placen-
\end{abstract}

tas have a significantly higher CDR compared to uncomplicated monochorionic twin placentas. Large inter-twin $\mathrm{Hb}$ differences in TAPS are associated with higher CDR.

(C) 2016 The Author(s)

Published by S. Karger AG, Basel

\section{Introduction}

Twin anemia-polycythemia sequence (TAPS) is a recently described form of chronic feto-fetal transfusion, characterized by large inter-twin hemoglobin $(\mathrm{Hb})$ differences, without signs of twin-twin transfusion syndrome (TTTS) [1]. TAPS may occur spontaneously in $3-5 \%$ of monochorionic pregnancies (spontaneous TAPS) [2] or after laser surgery for TTTS in $2-13 \%$ of TTTS cases (post-laser TAPS) [3,4]. The pathogenesis of TAPS is based on the presence of few, minuscule arteriovenous anastomoses allowing a slow transfusion of blood from the donor to the recipient and leading gradually to discordant $\mathrm{Hb}$ levels [1]. The postnatal criteria for TAPS are based on inter-twin $\mathrm{Hb}$ difference $>8 \mathrm{~g} / \mathrm{dl}$ and at least on one of the following: reticulocyte count ratio $>1.7$ and/ or presence of few minuscule placental vascular anastomoses determined through color dye injection [5]. However, the reticulocyte count ratio is not always measured

\section{KARGER}

E-Mail karger@karger.com www.karger.com/fdt

\section{2016 The Author(s) \\ Published by S. Karger AG, Basel 1015-3837/16/0402-0123\$39.50/0}

This article is licensed under the Creative Commons AttributionNonCommercial-NoDerivatives 4.0 International License (CC BYNC-ND) (http://www.karger.com/Services/OpenAccessLicense). Usage and distribution for commercial purposes as well as any distribution of modified material requires written permission. 
Fig. 1. Maternal side of a TAPS placenta showing the difference in color between the plethoric share of the recipient (left side of the placenta) and anemic share of the donor (right side). Histograms show the color intensity from the 2 placenta shares and from the entire placenta.

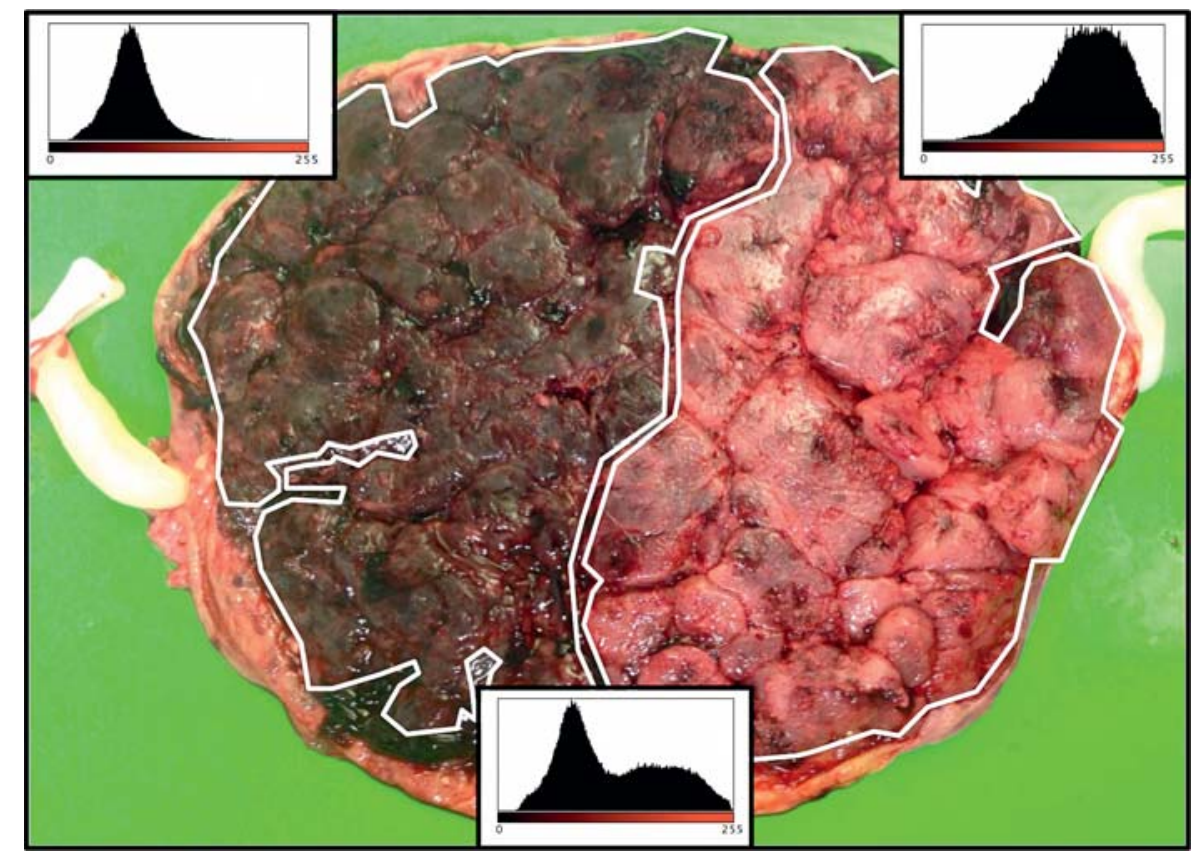

and placental injection is difficult to perform. This prompted us to search for an alternative diagnostic criterion that could be more practical and readily available for all the practitioners involved in the care of monochorionic twins throughout the world. Anecdotal reports have shown that the maternal side of TAPS placentas is also characterized by a striking difference in colors between the pale placental share of the anemic donor and the plethoric share of the recipient $[6,7]$. The difference in thickness and echodensity has also been noted antenatally during ultrasound examination [1].

The objective of this study is to determine the color difference between the 2 placental shares in a cohort of TAPS placentas compared to a control group of uncomplicated monochorionic placentas and to discuss the potential value of this new and alternative diagnostic criterion.

\section{Methods}

All monochorionic placentas examined at our center (Leiden University Medical Center) are routinely injected with colored dye and photographed according to a previously published protocol [7]. In the last 5 years, an additional evaluation was added to the protocol and included a routine picture of the maternal side of the placenta. For this study, we included all TAPS placentas evaluated at our center in which a clear digital picture of the maternal side of the placenta was recorded. All pictures were taken under the same (light) conditions. Each TAPS placenta (spontaneous TAPS and post-laser TAPS) was compared with a consecutive control placenta from uncomplicated monochorionic twin pregnancies, in which a digital picture of the maternal side was taken. TAPS was diagnosed using the previously described postnatal criteria: an inter-twin $\mathrm{Hb}$ difference $>8 \mathrm{~g} / \mathrm{dl}$ and at least a reticulocyte count $>1.7$ or small vascular anastomoses (diameter $<1 \mathrm{~mm}$ ) at the placental surface [5].

The obstetric and neonatal data were retrieved from our database, including gender, gestational age at delivery, type of delivery, $\mathrm{Hb}$ levels and reticulocyte count at birth. For the purpose of this study, we excluded placentas with insufficient picture quality and placentas with incomplete data.

The primary outcome of this study was the color difference ratio (CDR) between the 2 placental shares of the maternal side of each placenta from TAPS or uncomplicated monochorionic twins. The CDR was measured using a freely available image processing program called ImageJ version 1.48 (http://imagej.nih.gov/ij/ download.html). The digital picture of the placenta was first converted to a red spectrum channel in order to measure the intensity of the red (placenta) color. Measurement areas of the placenta share of each twin were selected manually. Two color intensity histograms of the different placental shares were then obtained. The $\mathrm{x}$-axis of the color intensity histogram displays a continuous red color scale (with values ranging from 0 to 255), whereas the y-axis displays the amount of pixels corresponding with that particular shade of red. A peak on the intensity histogram reflects the mode, which represents the color that is most present in the area selected. CDR is determined by dividing the mode of the color intensity histogram with the higher peak by the mode of the color intensity histogram with lower peak. A step-by-step tutorial on measuring CDR can be watched at https://www.youtube.com/watch?v= OSd6utv2Bw. An example of CDR measurement and matching color intensity histograms are depicted in figure 1 . 
Table 1. Baseline characteristics in monochorionic twin pairs with spontaneous TAPS and uncomplicated monochorionic twin pairs

\begin{tabular}{lcc}
\hline & $\begin{array}{l}\text { TAPS group } \\
(\mathrm{n}=19)\end{array}$ & $\begin{array}{l}\text { Control group } \\
(\mathrm{n}=19)\end{array}$ \\
\hline Female & $7 / 19(37)$ & $9 / 19(47)$ \\
Gestational age at birth, weeks & $33(30-35)$ & $36(33-37)$ \\
Cesarean delivery & $11 / 19(58)$ & $8 / 19(42)$ \\
Birth weight, g & $1,790(1,500-2,350)$ & $2,222(1,575-2,750)$ \\
\hline
\end{tabular}

Data are displayed as median (IQR) or n/N (\%).

\section{Statistics}

Continuous variables were compared using an independent sample t test. The Spearman rank correlation test was used to study the association between the inter-twin $\mathrm{Hb}$ differences and CDR in the TAPS group and control group. A p value $<0.05$ was considered to indicate statistical significance. Statistical analysis was performed using IBM SPSS Statistics version 20.0 (SPSS Inc., an IBM Company, Chicago, Ill., USA).

\section{Results}

A total of 22 TAPS placentas were eligible for this study. We excluded 3 placentas due to an insufficient picture quality $(\mathrm{n}=2)$ and incomplete data $(\mathrm{n}=1)$. A total of 19 TAPS placentas and 19 uncomplicated monochorionic placentas were analyzed.

The TAPS group consisted of 12 spontaneous TAPS placentas (63\%) and 7 post-laser TAPS placentas (37\%). The median gestational age at delivery was 33 weeks (interquartile range [IQR] 30-35 weeks) in the TAPS group and 36 (IQR 33-37 weeks) in the control group. Further details on the baseline characteristics are shown in table 1 .

Details on the CDR and hematological characteristics in the TAPS group and the control group are shown in table 2. An inter-twin $\mathrm{Hb}$ difference $>8 \mathrm{~g} / \mathrm{dl}$ was found in all TAPS twin pairs (median 12.7; range 10.0-21.8) and in none of the uncomplicated monochorionic twin pairs (median 1.6; range 0.2-7.7). Reticulocyte count ratio was measured in $84 \%$ (16 of 19) of the TAPS twin pairs (median 2.8; range 1.0-6.1) and in 100\% (19 of 19) of the uncomplicated monochorionic twin pairs (median 1.1; range 0.7-1.6). The median CDR in the group with and without TAPS was 2.73 (range 1.73-6.36) and 1.09 (range $1.00-1.35)$, respectively $(\mathrm{p}<0.01)$.

Figure 2 shows the CDRs in both TAPS and uncomplicated monochorionic twin placentas. All TAPS placen-
Table 2. Hematological differences and placenta share color difference between monochorionic twin pairs with TAPS and uncomplicated monochorionic twin pairs

\begin{tabular}{lccl}
\hline & $\begin{array}{l}\text { TAPS group } \\
(\mathrm{n}=19)\end{array}$ & $\begin{array}{l}\text { Control group } \\
(\mathrm{n}=19)\end{array}$ & $\begin{array}{l}\mathrm{p} \\
\text { values }\end{array}$ \\
\hline Inter-twin Hb difference, g/dl & $12.7(11.5-17.5)$ & $1.6(0.6-5.7)$ & $<0.01$ \\
Reticulocyt count ratio & $3.8(2.6-4.9)$ & $1.1(1.0-1.2)$ & $<0.01$ \\
CDR & $2.74(2.05-4.13)$ & $1.09(1.01-1.21)$ & $<0.01$ \\
\hline
\end{tabular}

Data are shown as median (IQR).

tas had a CDR value $>1.5$, whereas none of the CDR values of the control group was $>1.5$. CDR measurements and inter-twin $\mathrm{Hb}$ difference values were positively correlated (Spearman correlation coefficient $=0.83, \mathrm{p}<$ 0.01 ). This positive correlation was significant in the TAPS group $(\mathrm{R}=0.66, \mathrm{p}<0.01)$ but not in the control group $(\mathrm{R}=0.04, \mathrm{p}=0.87)$.

\section{Discussion}

This study shows that TAPS placentas are characterized by a higher CDR compared to uncomplicated monochorionic twin placentas. Similar to the color of the neonates at birth, the placental share of the recipient twin in TAPS is typically dark and plethoric and the placental share of the donor twin is pale and anemic. In this first pilot study of 38 placentas, all TAPS placentas had a CDR $>1.5$ whereas the CDR in the control group of uncomplicated monochorionic placentas was always $<1.5$, suggesting that a cutoff of 1.5 could be useful to differentiate both groups. In addition, we found a positive correlation between CDR and inter-twin $\mathrm{Hb}$ difference, suggesting a strong association between larger inter-twin $\mathrm{Hb}$ difference and higher CDR. However, this correlation was only present in the TAPS group and not in the control group of monochorionic twins. Whether measurements of CDR in placentas can be added as a new diagnostic criterion to the list of postnatal diagnostic criteria for TAPS requires further investigation.

The current postnatal diagnostic criteria for TAPS are based on the presence of an inter-twin $\mathrm{Hb}$ difference $>8 \mathrm{~g} / \mathrm{dl}$ and at least on one of the following: a reticulocyte count ratio $>1.7$ and/or the presence of small anastomoses (diameter $<1 \mathrm{~mm}$ ) on the placental surface [5]. Unfortunately, reticulocyte count measurements are sometimes omitted, and placental injection is not easy to per- 
Fig. 2. CDR versus inter-twin $\mathrm{Hb}$ difference in the TAPS group and control group of uncomplicated monochorionic twin placentas. Correlation between CDR and inter-twin $\mathrm{Hb}$ difference in the TAPS group and control group is $\mathrm{R}=0.66(\mathrm{p}<$ $0.01)$ and $R=0.04(p=0.87)$, respectively.

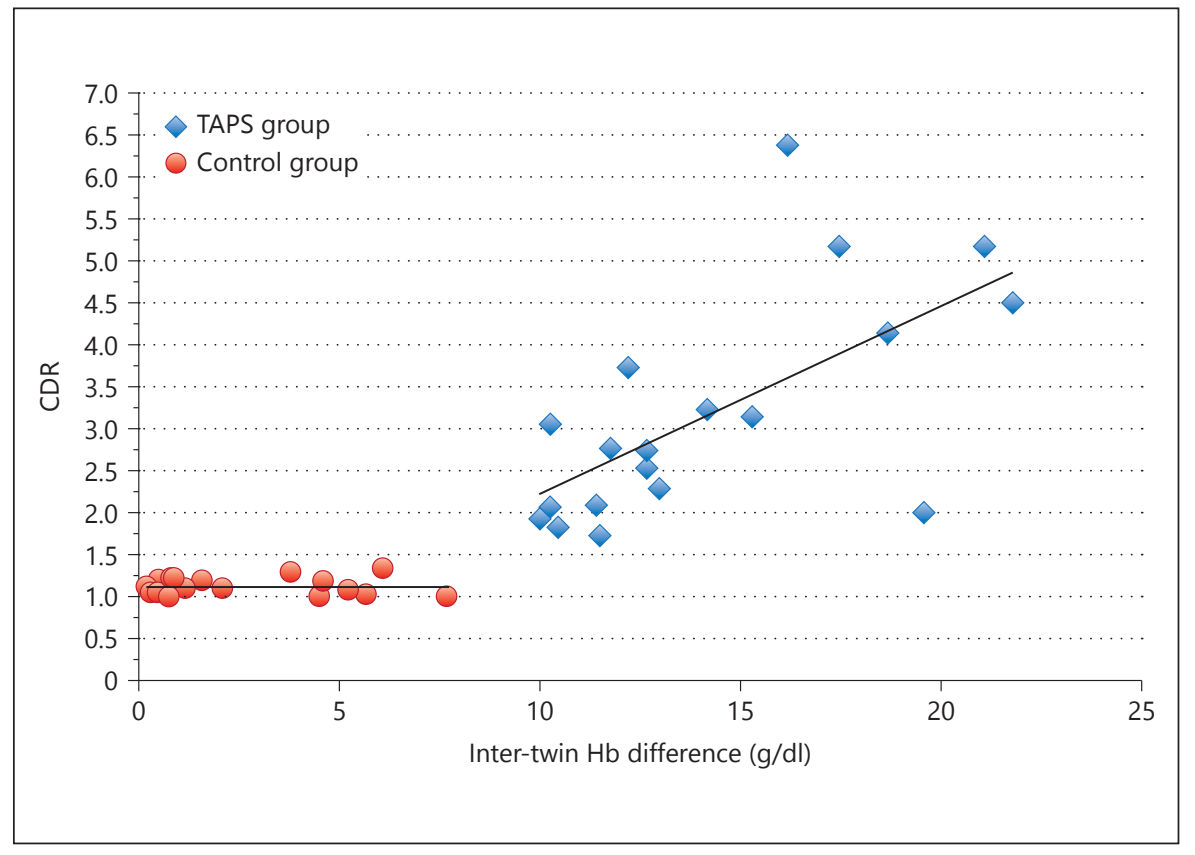

form and therefore only performed in a few specialized centers. In addition, evaluation of the fetal side of the placenta may sometimes be hampered by leakage of color dye or damage of the placental vessels preventing accurate evaluation of the vascular anastomoses. As shown in this study, measurements of the color difference of the maternal side of the placenta using a freely available image processing program, ImageJ, could prove to be of additional value in the diagnosis of TAPS. These measurements made using a digital picture of the placenta were quick and easy to perform. Some important technical specifications are required to perform accurate measurements, such as ensuring that blood clots on the maternal side of the placentas are washed out before taking the picture and avoiding direct light shining on the placentas to prevent reflections.

Whether this method can eventually be added to the list of postnatal criteria requires further investigations in larger series of placentas with and without TAPS to determine the sensitivity and specificity of the test. Importantly, CDR measurements should also be investigated in placentas with acute TTTS to determine whether this method can help distinguish TAPS cases from cases with acute peripartum TTTS. In both situations, large inter-twin Hb differences are present at birth [5]. However, inter-twin transfusion in TAPS is a chronic process leading to chronic anemia in the donor (reflected by the presence of increased reticulocyte count) and chronic polycythemia in the re- cipient [5]. In contrast, inter-twin transfusion in acute peripartum TTTS is an acute event leading to acute anemia in the donor and hypovolemic shock in case of massive blood loss and acute polycythemia in the recipient [8]. Neonatal management in both situations requires a different approach with acute interventions in acute TTTS (including acute blood transfusion in the donor) [8] in contrast to a more conservative approach in case of chronic transfusion in TAPS [1]. In the future, whether the examination of the maternal side of the placenta will help perinatologists to make a quick distinction between acute peripartum TTTS and TAPS remains to be established.

In conclusion, this new and promising method shows that TAPS placentas have a large color difference compared to placentas from uncomplicated monochorionic twins. However, our data should be interpreted with care due to the retrospective nature of the study and the relatively small sample size. Further investigations are needed to determine the sensitivity and specificity of this test and to examine whether measurement of color difference would also help in distinguishing between TAPS and acute peripartum TTTS.

\section{Acknowledgments}

The authors thank Dirk Dokter from Delft University of Technology for his invaluable technical advice. 


\section{References}

$>$ Slaghekke F, Kist WJ, Oepkes D, Pasman SA, Middeldorp JM, Klumper FJ, Walther FJ, Vandenbussche FP, Lopriore E: Twin anemia-polycythemia sequence: diagnostic criteria, classification, perinatal management and outcome. Fetal Diagn Ther 2010;27:181-190.

-2 Gucciardo L, Lewi L, Vaast P, Debska M, De Catte L, Van Mieghem T, Done E, Devlieger $\mathrm{R}$, Deprest J: Twin anemia polycythemia sequence from a prenatal perspective. Prenat Diagn 2010;30:438-442.

3 Habli M, Bombrys A, Lewis D, Lim FY, Polzin $\mathrm{W}$, Maxwell R, Crombleholme T: Incidence of complications in twin-twin transfusion syndrome after selective fetoscopic laser photocoagulation: a single-center experience. Am J Obstet Gynecol 2009;201:417.e1-e7.
4 Robyr R, Lewi L, Salomon LJ, Yamamoto M, Bernard JP, Deprest J, Ville Y: Prevalence and management of late fetal complications following successful selective laser coagulation of chorionic plate anastomoses in twin-totwin transfusion syndrome. Am J Obstet Gynecol 2006;194:796-803.

5 Lopriore E, Slaghekke F, Oepkes D, Middeldorp JM, Vandenbussche FP, Walther FJ: Hematological characteristics in neonates with twin anemia-polycythemia sequence (TAPS). Prenat Diagn 2010;30:251255.
6 van Meir H, Slaghekke F, Lopriore E, van wijngaarden WJ: Arterio-arterial anastomoses do not prevent the development of twin anemia-polycythemia sequence. Placenta 2010; 31:163-165.

7 Lopriore E, Slaghekke F, Middeldorp JM, Klumper FJ, van Lith JM, Walther FJ, Oepkes D: Accurate and simple evaluation of vascular anastomoses in monochorionic placenta using colored dye. J Vis Exp 2011; 55:e3208.

8 Lopriore E, Holtkamp N, Sueters M, Middeldorp JM, Walther FJ, Oepkes D: Acute peripartum twin-twin transfusion syndrome: incidence, risk factors, placental characteristics and neonatal outcome. J Obstet Gynaecol Res 2014;40:18-24. 